\title{
SOLUTION OF NONLINEAR PARTIAL DIFFERENTIAL EQUATIONS BY TRIPLE LAPLACE DECOMPOSITION METHOD
}

Adil Mousa

Department of Mathematics

Faculty of Science \&Technology

Omdurman Islamic University, Khartoum, Sudan

\begin{abstract}
- in this work, we have discussed the solutions of partial differential equations nonlinear in Three Dimensions by Combining the Adomian decomposition method and triple Laplace transform and the examples application to solve partial differential equations in threedimensions.
\end{abstract}

Keywords-Triple Laplace transform, Inverse Triple Laplace transform, Adomian decomposition method.

\section{INTRODUCTION}

The method was developed from 1970 to the 1990 by George Adomian the chair of the center for applied mathematics at the university of Georgia in (USA) is called Adomian decomposition method (ADM) is a semi analytical method for solving ordinary and partial nonlinear differential equations.[1-6]

P. S. Laplace (1749-1827) introduced the idea of the Laplace transform in 1782: [7-10] The Laplace transform denoted by the operator $L$ is defined as

$$
L[f(t): \rho)]=\int_{0}^{\infty} e^{-\rho t} f(t) d t, t>0
$$

The main objective of the study is to extend the method of decomposition algorithm to convert one-dimensional Laplace Adomian decomposition method to threedimensional Laplace Adomian decomposition method to solve nonlinear partial differential equations. The advantage of this method is Its capability to combining two powerful methods to obtain accurate solutions to nonlinear threedimensional equations, Several examples are given to reestablish the effectiveness of this method. [11-13]

\section{Proposed Algorithm}

\section{A. Theorems and Definitions of triple Laplace transform}

Definition: 1 Let $f(x, y, t)$ be a function that can be

expressed as convergent infinite series, and let $(x, y, t) \in R_{3}^{+}$, then, the triple Laplace Transform is denoted by:

\author{
Tarig M. Elzaki
}

Mathematics Department

Faculty of Sciences and Arts-Alkamil

University of Jeddah, Jeddah-Saudi Arabia

$L_{3}[f(x, y, t):(\sigma, \rho, \delta)]=$

$\int_{0}^{\infty} \int_{0}^{\infty} \int_{0}^{\infty} e^{-\sigma x-\rho y-\delta t} f(x, y, t) d x d y d t$

where $x, y, t>0$ and $\sigma, \rho, \delta$ are Laplace variables, and

$$
\begin{aligned}
f(x, y, t) & =\frac{1}{2 \pi i} \int_{\alpha-i \infty}^{\alpha+i \infty} e^{\sigma x}\left[\frac{1}{2 \pi i} \times\right. \\
& \left.\int_{\beta-i \infty}^{\beta+i \infty} e^{\rho y}\left[\frac{1}{2 \pi i} \int_{\lambda-i \infty}^{\lambda+i \infty} e^{\delta t} f(\sigma, \rho, t) d \delta\right] d \rho\right] d \sigma
\end{aligned}
$$

is the inverse triple Laplace transform denoted by $L_{3}^{-1}$.

Theorem: 2 let $f(t)$ be in $S$ and let $F_{n}(\rho)$ denote Laplace transform of nth derivative, $f^{n}(t)$ of $f(t)$, then for $n \geq 1$

$$
F_{n}(\rho)=\rho^{n} F(\rho)-\sum_{k=0}^{n-1} \rho^{n-1-k} f^{(k)}(0)
$$

To obtain Laplace transform of partial derivative we use integration by parts, and then we have $L_{3}\left[\frac{\partial f(x, y, t)}{\partial t}\right]=\delta F(\sigma, \rho, \delta)-F(\sigma, \rho, 0)$,

$$
L_{3}\left[\frac{\partial^{2} f(x, y, t)}{\partial t^{2}}\right]=\delta^{2} F(\sigma, \rho, \delta)-\delta F(\sigma, \rho, 0)-\frac{\partial F(\sigma, \rho, 0)}{\partial t},
$$$$
L_{3}\left[\frac{\partial^{3} f(x, y, t)}{\partial x \partial y \partial t}:(\sigma, \rho, \delta)\right]=\sigma \rho \delta F(\sigma, \rho, \delta)+\sigma F(\sigma, 0,0)+
$$

$$
\rho F(0, \rho, 0)+\delta F(0,0, \delta)-\sigma \rho F(\sigma, \rho, 0)-
$$

$\sigma \delta F(\sigma, 0, \delta)-\rho \delta F(0, \rho, \delta)-F(0,0,0)$

\section{$B$. Theorems and properties of triple Laplacetransform \\ Decomposition Method for PDEs -}

In this section, we to illustrate the basic idea of this method, we consider a general non-linear no homogeneous partial differential equation

$$
\frac{\partial^{m} u(x, y, t)}{\partial t^{m}}+R u(x, y, t)+N u(x, y, t)=g(x, y, t),
$$


Where $m=1,2,3$, with the initial conditions

$\left.\frac{\partial^{m-1} u(x, y, t)}{\partial t^{m-1}}\right|_{t=0}=f_{m-1}(x, y), m=1,2,3$,

where $\frac{\partial^{m} u(x, y, t)}{\partial t^{m}}$ is the partial derivative of the function $u(x, y, t)$ of order $m(m=1,2,3),, \quad R \quad$ is the linear differential operator, $N$ represents the general nonlinear differential operator, and $g(x, y, t)$ is the source term. Applying the triple Laplace transform (denoted in this paper by $L_{3}$ ) on both sides of Eq. (5), we get

$L_{3}\left[\frac{\partial^{m} u(x, y, t)}{\partial t^{m}}\right]+L_{3}[R u(x, y, t)]+$

$L_{3}[N u(x, y, t)]=L_{3}[g(x, y, t)]$,

Using the properties of Laplace transform, we obtain

$\delta^{m} L_{3}[u(x, y, t)]=\sum_{k=0}^{m-1} \delta^{m-1-k} \frac{\partial^{k} u(x, y, 0)}{\partial t^{k}}+$

$L_{3}[g(x, y, t)]-L_{3}[R u(x, y, t)+N u(x, y, t)]$,

Where $m=1,2,3$, and thus, we have

$$
L_{3}[u(x, y, t)]=\sum_{k=0}^{m-1} \delta^{-1-k} \frac{\partial^{k} u(x, y, 0)}{\partial t^{k}}+
$$

$\delta^{-m} L_{3}[g(x, y, t)]-\delta^{-m} L_{3}[R u(x, y, t)+N u(x, y, t)]$,

Operating the inverse transform on both sides of Eq. (9), we get

$u(x, y, t)=G(x, y, t)-$

$L_{3}^{-1}\left(\delta^{-m} E[R u(x, y, t)+N u(x, y, t)]\right)$,

where $G(x, y, t)$, represents the term arising from the source term and the prescribed initial conditions.

The second step in Laplace transform Decomposition Method, is that we represent the solution as an infinite series given below

$u(x, y, t)=\sum_{n=0}^{\infty} u_{n}(x, y, t)$

and the nonlinear term can be decomposed as

$N u(x, y, t)=\sum_{n=0}^{\infty} A_{n}$,

where $A_{n}$ are Adomian polynomials of $u_{0}, u_{1}, u_{2}, \cdots u_{n}$ and it can be calculated by the formula given below

$A_{n}=\frac{1}{n !} \frac{\partial^{n}}{\partial \lambda^{n}}\left[N \sum_{i=0}^{\infty} \lambda^{i} u_{i}\right]_{\lambda=0}, n=0,1,2,3, \ldots$

Substituting (11) and (12) in (10), we have

$$
\sum_{n=0}^{\infty} u_{n}(x, y, t)=G(x, y, t)-L^{-1}\left(\delta^{-m} L\left[R \sum_{n=0}^{\infty} u_{n}+\sum_{n=0}^{\infty} A_{n}\right]\right)
$$

On comparing both sides of the Eq. (14), we get

$$
\begin{array}{r}
u_{0}(x, y, t)=G(x, y, t), \\
u_{1}(x, y, t)=-L^{-1}\left(\delta^{-m} L\left[R u_{0}(x, y, t)+A_{0}\right]\right), \\
u_{2}(x, y, t)=-L^{-1}\left(\delta^{-m} L\left[R u_{1}(x, y, t)+A_{1}\right]\right), \\
u_{3}(x, y, t)=-L^{-1}\left(\delta^{-m} L\left[R u_{2}(x, y, t)+A_{2}\right]\right),
\end{array}
$$

In general, the recursive relation is given as

$u_{n+1}(x, y, t)=-L^{-1}\left(\delta^{-m} L\left[R u_{n}(x, y, t)+A_{n}\right]\right)$,

where $m=1,2,3$, and $n \geq 0$,

Finally, we approximate the analytical solution $u(x, y, t)$ by truncated series

$u(x, y, t)=\lim _{\aleph \rightarrow \infty} \sum_{n=0}^{\aleph} u_{n}(x, y, t)$

\section{EXPERIMENT AND RESULT}

In this section, we apply triple Laplace decomposition method for PDEs to solve nonlinear partial differential equations of the three-dimensional.

Example 1: Consider nonlinear partial differential equation $\mathrm{u}_{t}(x, y, t)+6 \mathrm{u}(x, y, t) \mathrm{u}_{x y}(x, y, t)+\mathrm{u}_{x x}(x, y, t)=0$

with initial conditions $\mathrm{u}(\mathrm{x}, y, 0)=\mathrm{xy}$

Solution: Let us rewrite Equation (18) as:

$\mathrm{u}_{t}(x, y, t)=\left[-6 \mathrm{u}(x, y, t) \mathrm{u}_{x y}(x, y, t)-\mathrm{u}_{x x}(x, y, t)\right]$

By taking the Laplace transform to both sides

$\mathrm{L}_{3}\left[\mathrm{u}_{t}(x, y, t)\right]=\mathrm{L}_{3}\left[-6 \mathrm{u}(x, y, t) \mathrm{u}_{x y}(x, y, t)-\mathrm{u}_{x x}(x, y, t)\right]$

(19)

Recall that $\quad \mathrm{L}_{3}\left[\mathrm{u}_{t}\right]=\delta F(\sigma, \rho, \delta)-F(\sigma, \rho, 0)$

So Equation (19) becomes;

$\delta F(\sigma, \rho, \delta)-F(\sigma, \rho, 0)=$

$L_{3}\left[-6 \mathrm{u}(x, y, t) \mathrm{u}_{x y}(x, y, t)-\mathrm{u}_{x x}(x, y, t)\right]$

(20)

A taking the given initial conditions on Equation (20) and simplifying, we obtain;

$T(\sigma, \rho, \delta)=\frac{1}{\sigma^{2} \rho^{2} \delta}-\frac{1}{\delta} L_{3}\left[6 \mathrm{u}(x, y, t) \mathrm{u}_{x y}(x, y, t)+\mathrm{u}_{x x}(x, y, t)\right]$

A taking the inverse triple Laplace transforms to Equation (21), we have;

$$
\begin{aligned}
& u(x, y, t)=L_{3}^{-1}\left[\frac{1}{\sigma^{2} \rho^{2} \delta}\right]- \\
& L_{3}^{-1}\left\{\frac{1}{\delta} L_{3}\left[6 \mathrm{u}(x, y, t) \mathrm{u}_{x y}(x, y, t)+\mathrm{u}_{x x}(x, y, t)\right]\right\}
\end{aligned}
$$

The resulting expression is

$u(x, y, t)=x y-$ 
$L_{3}^{-1}\left\{\frac{1}{\delta} \mathrm{L}_{3}\left[6 \mathrm{u}(x, y, t) \mathrm{u}_{x y}(x, y, t)+\mathrm{u}_{x x}(x, y, t)\right]\right\}$

From Equation (22) let $u_{0}(x, y, t)=x y$

The recursive relation is given as:

$u_{n+1}(x, y, t)=-L_{3}^{-1}\left\{\frac{1}{\delta} \mathrm{L}_{3}\left[6 \mathrm{~A}_{n}+\frac{\partial^{2} \mathrm{u}_{n}(x, y, t)}{\partial x^{2}}\right]\right\}$

Note that $A_{n}$ is the Adomian polynomial to decompose the nonlinear terms by using the relation:

$A_{n}=\frac{1}{n !} \frac{d^{n}}{d \lambda^{n}} f\left[\sum_{i=0}^{\infty} \lambda^{i} u_{i}(x, y, t)\right]_{\lambda=0}$

Let the nonlinear term be represented

$f[u(x, y, t)]=u(x, y, t) \frac{\partial^{2} u(x, y, t)}{\partial x \partial y}$

By using Equation (25) in Equation (24), we obtain;

$$
\begin{aligned}
A_{0}= & u_{0}(x, y, t) \frac{\partial^{2} u_{0}(x, y, t)}{\partial x \partial y}, \\
A_{1}= & u_{1}(x, y, t) \frac{\partial^{2} u_{0}(x, y, t)}{\partial x \partial y}+u_{0}(x, y, t) \frac{\partial^{2} u_{1}(x, y, t)}{\partial x \partial y}, \\
A_{2}= & u_{2}(x, y, t) \frac{\partial^{2} u_{0}(x, y, t)}{\partial x \partial y}+ \\
& u_{1}(x, y, t) \frac{\partial^{2} u_{1}(x, y, t)}{\partial x \partial y}+u_{0}(x, y, t) \frac{\partial^{2} u_{2}(x, y, t)}{\partial x \partial y}, \ldots
\end{aligned}
$$

From Equation (23)

When $\mathrm{n}=0$, we have

$u_{1}(x, y, t)=-L_{3}^{-1}\left\{\frac{1}{\delta} L_{3}\left[6 \mathrm{~A}_{0}+\frac{\partial^{2} \mathrm{u}_{0}(x, y, t)}{\partial x^{2}}\right]\right\}$

is computed as: $A_{0}=x y$

$u_{1}(x, y, t)=-L_{3}^{-1}\left\{\frac{1}{\delta} L_{3}\left[6 x y+\frac{\partial^{2}}{\partial x^{2}}[x y]\right]\right\}$

By simplifying $u_{1}(x, y, t)=-6 x y t$

When $\mathrm{n}=1$, we have;

$u_{2}(x, y, t)=-L_{3}^{-1}\left\{\frac{1}{\delta} L_{3}\left[6 \mathrm{~A}_{1}+\frac{\partial^{2} \mathrm{u}_{1}(x, y, t)}{\partial x^{2}}\right]\right\}$

is computed as: $A_{1}=-12 x y t$

$u_{2}(x, y, t)=-L_{3}^{-1}\left\{\frac{1}{\delta} L_{3}\left[-72 x y t+\frac{\partial^{2}}{\partial x^{2}}[-6 x y t]\right]\right\}$

By simplifying $u_{2}(x, y, t)=36 x y t^{2}$

The approximate series solution is

$u(x, y, t)=x y-6 x y t+36 x y t^{2}-216 x y t^{3}+\ldots$

This can be written as $u(x, y, t)=x y\left[1-6 t+[6 t]^{2}-[6 t]^{3}+\ldots\right]$

By using Taylor's series, the closed form solution will be as follows

$u(x, y, t)=\frac{x y}{1+6 t}$

Example 2: Consider nonlinear partial differential equation

$$
\mathrm{u}_{y}(x, y, t) \mathrm{u}_{t}(x, y, t)-\mathrm{u}_{x x}(x, y, t)=\mathrm{u}(x, y, t)
$$

with initial conditions

$\mathrm{u}(0, y, t)=\mathrm{yt}, \mathrm{u}_{x}(0, y, t)=-1$

Solution: Let us rewrite Equation (26) as:

$\mathrm{u}_{x x}(x, y, t)=\mathrm{u}_{y}(x, y, t) \mathrm{u}_{t}(x, y, t)-\mathrm{u}(x, y, t)$

By taking the triple Laplace transform to both sides $\mathrm{L}_{3}\left[\mathrm{u}_{x x}(x, y, t)\right]=\mathrm{L}_{3}\left[\mathrm{u}_{y}(x, y, t) \mathrm{u}_{t}(x, y, t)-\mathrm{u}(x, y, t)\right]$

Recall that

$\mathrm{L}_{3}\left[\mathrm{u}_{x x}(x, y, t)\right]=\sigma^{2} F(\sigma, \rho, \delta)-\sigma F(0, \rho, \delta)-\frac{\partial F(0, \rho, \delta)}{\partial x}$

So Equation (27) becomes;

$\sigma^{2} F(\sigma, \rho, \delta)-\sigma F(0, \rho, \delta)-\frac{\partial F(0, \rho, \delta)}{\partial x}=$

$L_{3}\left[\mathrm{u}_{y}(x, y, t) \mathrm{u}_{t}(x, y, t)-\mathrm{u}(x, y, t)\right]$

(28)

taking the given initial conditions on Equation (28) and simplifying, we obtain;

$$
\begin{aligned}
& F(\sigma, \rho, \delta)=\frac{1}{\sigma \rho^{2} \delta^{2}}-\frac{1}{\sigma^{2} \rho \delta}- \\
& \frac{1}{\sigma^{2}} L_{3}\left[\mathrm{u}(x, y, t)-\mathrm{u}_{y}(x, y, t) \mathrm{u}_{t}(x, y, t)\right]
\end{aligned}
$$

A taking the inverse triple Laplace transform to Eq. (29), we have;

$$
\begin{aligned}
& u(x, y, t)=L_{3}^{-1}\left[\frac{1}{\sigma \rho^{2} \delta^{2}}-\frac{1}{\sigma^{2} \rho \delta}\right]- \\
& L_{3}{ }^{-1}\left\{\frac{1}{\sigma^{2}} \mathrm{~L}_{3}\left[\mathrm{u}(x, y, t)-\mathrm{u}_{y}(x, y, t) \mathrm{u}_{t}(x, y, t)\right]\right\}
\end{aligned}
$$

The resulting expression is

$u(x, y, t)=y t-x-$

$L_{3}^{-1}\left\{\frac{1}{\sigma^{2}} \mathrm{~L}_{3}\left[\mathrm{u}(x, y, t)-\mathrm{u}_{y}(x, y, t) \mathrm{u}_{t}(x, y, t)\right]\right\}$

From Equation (30) let $u_{0}(x, y, t)=y t-x$

The recursive relation is given as:

$u_{n+1}(x, y, t)=-L_{3}^{-1}\left\{\frac{1}{\sigma^{2}} L_{3}\left[\mathrm{~A}_{n}-\frac{\partial \mathrm{u}_{n}(x, y, t)}{\partial y} \frac{\partial \mathrm{u}_{n}(x, y, t)}{\partial t}\right]\right\}$ 
Note that $A_{n}$ is the Adomian polynomial to decompose the nonlinear terms by using the relation:

$A_{n}=\frac{1}{n !} \frac{d^{n}}{d \lambda^{n}} f\left[\sum_{i=0}^{\infty} \lambda^{i} u_{i}(x, y, t)\right]_{\lambda=0}$

Let the nonlinear term be represented

$$
f[u(x, y, t)]=u(x, y, t)
$$

By using Equation (33) in Equation (32), we obtain;

$A_{0}=u_{0}(x, y, t)$,

$A_{1}=u_{1}(x, y, t)+u_{0}(x, y, t)$,

$A_{2}=u_{2}(x, y, t)+u_{1}(x, y, t)+u_{0}(x, y, t), \ldots$

From equation (31), when $\mathrm{n}=0$, we have:

$u_{1}(x, y, t)=-L_{3}^{-1}\left\{\frac{1}{\sigma^{2}} L_{3}\left[\mathrm{~A}_{0}-\frac{\partial \mathrm{u}_{0}(x, y, t)}{\partial y} \frac{\partial \mathrm{u}_{0}(x, y, t)}{\partial t}\right]\right\}$

is computed as: $A_{0}=y t-x$

$u_{1}(x, y, t)=-L_{3}^{-1}\left\{\frac{1}{\sigma^{2}} L_{3}\left[[y t-x]-\frac{\partial[y t-x]}{\partial y} \frac{\partial[y t-x]}{\partial t}\right]\right\}$

By simplifying $u_{1}(x, y, t)=\frac{x^{3}}{3 !}$

The approximate series solution is

$u(x, y, t)=y t-x+\frac{x^{3}}{3 !}-\frac{x^{5}}{5 !}-\frac{1}{2} x^{2} y t+\frac{x^{3}}{3 !} \ldots$

This can be written as

$u(x, y, t)=y t-\left[x-\frac{x^{3}}{3 !}+\frac{x^{5}}{5 !}-\ldots\right]$

By using Taylor's series, the closed form solution will be as follows

$u(x, y, t)=y t-\sin x$

\section{CONCLUSION}

This work discussed the definition of the triple Laplace transform that was applied Some important theorems and properties have been presented for this relatively new transformation to find solutions for partial differential equations in three dimensions under the initial conditions, the triple Laplace transform study succeeded in achieving solutions.

\section{REFERENCE}

[1] Abdul Majid Wazwaz, Partial Differential Equations and Solitary Waves Theory, Higher Education Press Beijing and Springer - Verlag Berlin Heidelberg (2009).

[2] Moghadam MM, Saeedi $H$ Application of differential transforms for solving the Volterra integro-partial differential equations. Iranian Journal of Science and Technology, Transaction A 34(A1):59 (2010).
[3] Garci'a-Olivares, A., Analytic solution of partial differential equations with Adomian's decomposition, Kybernetes, 32 (3) 354-368 (2003).

[4] Tarig M. Elzaki , Adil Mousa On the convergence of triple Elzaki transform SN Applied Sciences 1:275https://doi.org/10.1007/s42452-019-0257-2 (2019)

[5] H. Eltayeb, A. Kilicman and S. Mesloub, Modi_ed Laplace decomposition method for solving system of equations Emden-Fowler type, Journal of Computational and Theoretical Nanoscience, 12 ,5297-5301, (2015).

[6] S.A. Khuri, A Laplace decomposition algorithm applied to a class of nonlinear deferential equations, J.Math. Annl. Appl., 4, 141-155, (2001).

[7] A. Khuri, A Laplace decomposition algorithm applied to a class of nonlinear differential equations, J. Math. Annl. Appl., 4, 141-155, (2001).

[8] Abdon Atangana A Note on the Triple Laplace Transform and Its Applications to Some Kind of Third-Order Differential Equation Abstract and Applied Analysis, Article ID 769102, 10 pages Volume (2013) .

[9] M. Khalid, M. Sultana, F. Zaidi and U. Arshad, An Elzaki Transform Decomposition Algorithm Applied to a Class of Non-Linear Diperential Equations, J. Natural Sci. Res., 5, 48-56, (2015).

[10] Trig M. Elzaki, Salih M. Elzaki, On connection between Laplace transform and Elzaki Transform, Advances in Theoretical and Applied Mathematics, ISSN0973-4554 volume 6 Number1, pp. 1-11, (211).

[11] M. M. Hosseini and H. Nasabzadeh, On the convergence of Adomian decomposition method, Appl. Math. Comput., 182, 536.543, (2006).

[12] D. Kumar, J. Singh and S. Rathore, Sumudu Decomposition Method for Nonlinear Equations, Int. Math. For., 7, 515 - 521.57-64, (2012).

[13] J.S. Duan and R. Rach, A new modification of the Adomian decomposition method for solving boundary value problems for higher order nonlinear differential equations, Appl. Math. Comput, 4090-4118, 218 (2011. 